\title{
RESONANT MICROCANTILEVER GAS SENSOR FABRICATED IN CMOS TECHNOLOGY FOR THE DETECTION OF CHEMICAL AGENTS
}

\author{
$\underline{\text { Ioana Voiculescu }}{ }^{1}$, Mona Zaghloul ${ }^{1}$, R. Andrew McGill ${ }^{2}$, Eric J. Houser ${ }^{2}$, Stanley Stepnowski ${ }^{2}$, Evgueni \\ Sokolovski ${ }^{2}$, Jennifer Stepnowski ${ }^{2}$, Joe Vignola ${ }^{2}$ and Gary K. Fedder ${ }^{3}$ \\ 1. George Washington University, Mechanical \& Aerospace Engineering, 801- $22^{\text {nd }}$ Street, NW, \\ Washington, DC 20052, phone: (571) 338-2048, fax: (202)767-5301, e-mail: ioana@gwu.edu \\ 2. Naval Research Laboratory, Washington, DC. 20375 \\ 3. Carnegie Mellon University, Dept. of Electrical \& Computer Engineering, Pittsburgh, PA. 15213
}

\begin{abstract}
The design, fabrication, and testing of a resonant cantilever in CMOS technology is presented in this paper. The new cantilever design described here includes interdigitated fingers for electrostatic actuation, and a piezoresistive Wheatstone bridge arrangement to read out the deflection signal. The reference resistors of the Wheatstone bridge are fabricated on auxiliary cantilevers that are immediately adjacent to the actuated device. The entire device is fabricated using a $0.6 \mu \mathrm{m}$ three metal, double poly CMOS (complementary metal oxide semiconductor) process, combined with subsequent micromachining steps. A thin polymer coating is applied to the surface of the microcantilever to provide a sorbent layer for chemical nerve agents. Exposing the sensor with the nerve agent simulant dimethylmethylphosphonate (DMMP), allowed demonstrated detection at a concentration of $20 \mathrm{ppb}$ or $0.1 \mathrm{mg} / \mathrm{m}^{3}$.
\end{abstract}

\section{INTRODUCTION}

A chemical sensor is a device, which converts analytic chemical information into a useful signal. Chemical sensors are important for a variety of industrial and environmental applications, including the detection of hazardous chemicals, quality control in the food, perfume, and beverage industries, and for medical applications.

A typical configuration for a chemical sensor includes a sorbent layer deposited on the active area of a transducer [1]. The interaction of a gas and the sorptive layer can be monitored as a function of physicochemical changes in the coating, and transduced into an electrical signal for ease of recording or display. The advent and maturation of microelectromechanical systems (MEMS) technology now offers many opportunities to dramatically reduce the size, cost, and power consumption of chemical sensors.

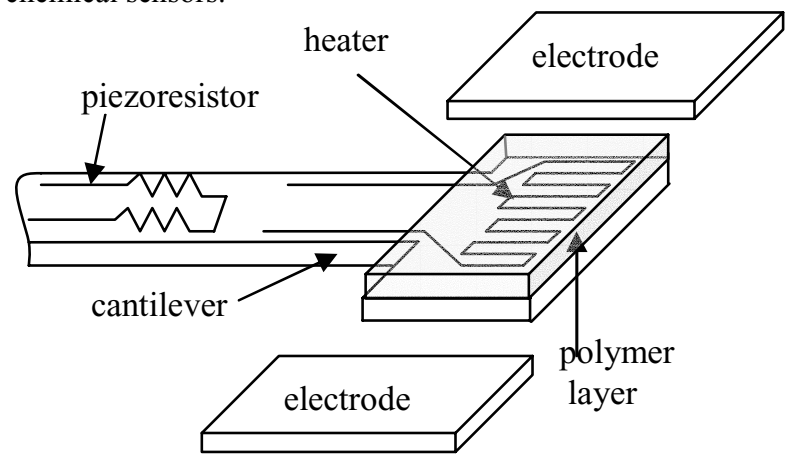

Figure 1. Microcantilever gas sensor. Electrodes are used for electrostatic actuation of the cantilever.

\section{SENSOR TECHNOLOGY AND DESCRIPTION}

A cantilever chemical sensor consists of two key components: a gas sorptive layer, such as a polymer, and the cantilever transducer as shown in Fig.1. In this design, only the cantilever tip is used for polymer coating. The uptake of different gases is monitored as a shift in the device frequency, which is reversible if the gas-polymer chemical interactions are reversible. The cantilever gas sensor is a resonating microbalance, with mass increases normally leading to a decrease in the cantilever resonance frequency. Electrostatic actuation is used to operate the cantilever in a resonant mode, and the resonance frequency is measured by a set of piezoresistors connected in a Wheatstone bridge configuration.

Piezoresistors

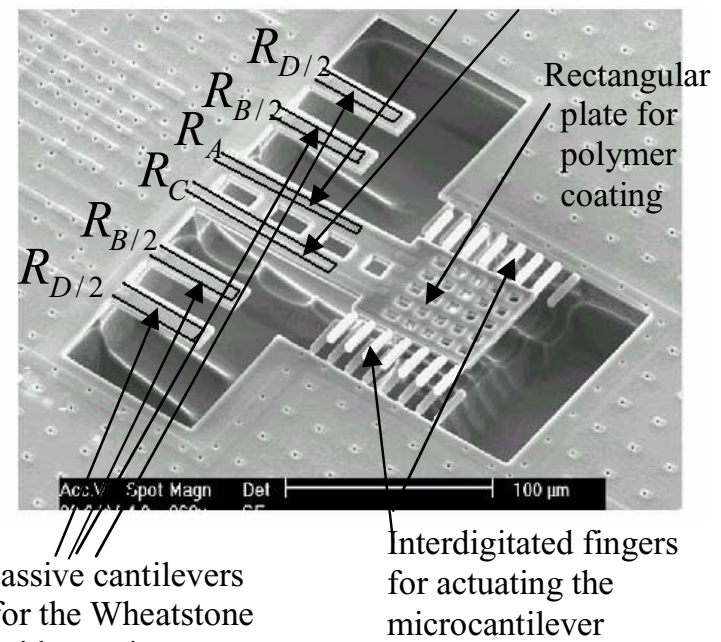

bridge resistors

Figure 2. SEM image of cantilever. $R_{A}, R_{B}, R_{C}$ and $R_{D}$ resistors are arranged in the on chip Wheatstone bridge.

The full Wheatstone bridge has two piezoresistors, $\mathrm{R}_{\mathrm{A}}$ and $\mathrm{R}_{\mathrm{C}}$, positioned on the active cantilever, and two reference resistors $\mathrm{R}_{\mathrm{B}}$ and $\mathrm{RD}$ situated on neighboring short, auxiliary cantilevers, as shown in Fig.2.

The chip was designed in CMOS-MEMS technology using MEMSCAP-Xplorer software installed under Cadence. The custom style used for the CMOS-MEMS process was developed at Carnegie Mellon University (CMU) [2]. Typical die size for this process is $2.5 \mathrm{~mm}$ by $2.5 \mathrm{~mm}$. The foundry used in this work was Austrian Microsystems (AMS) $(0.6 \mu \mathrm{m}, 3$-metal, 2-poly CMOS).

Travel support has been generously provided by the Transducers Research Foundation and by the DARPA MEMS and DARPA BioFlips programs. 
The standard CMOS-MEMS process was followed by two maskless dry etch steps to release the microstructures that are protected by the top-most metal layer [2].

\section{VIBRATION AMPLITUDE AND RESONANCE FREQUENCY}

The surface motion of the microcantilever oscillator was characterized with a scanning laser doppler vibrometer (LDV) system developed at the Naval Research Laboratory (NRL). The NRL LDV system has been previously described in detail [3]. The system illuminates the sample surface with light from an argon-ion laser (spot size $=2.5 \mu \mathrm{m}$ ). Scattered light for the sample surface is mixed on the surface of a photo detector with the frequency shifted, reference light. The photodetector produces a frequency modulated signal that, once demodulated, is proportional to the surface displacement at a single location. Fig. 3 shows the measured vibration amplitude at the tip of a bare microcantilever.

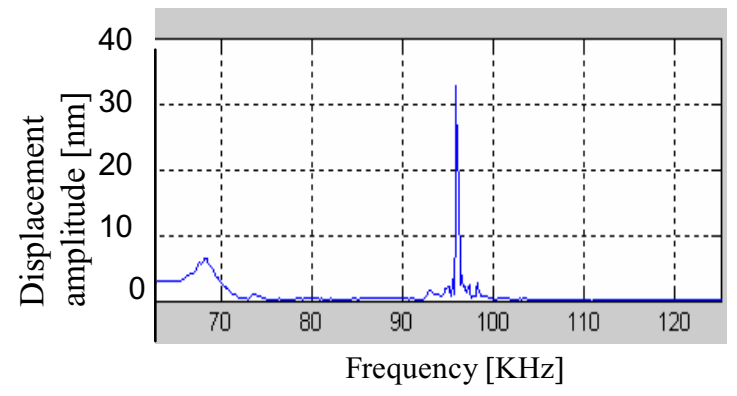

Figure 3. Vibration amplitude of the microcantilever as a function of frequency.

Using the identified fundamental resonance frequency, the cantilever chip was then characterized using a spectrum analyzer. The cantilever device was operated electrostatically by driving it with a small varying ac signal imposed on a constant dc bias. Breadboard electronics and a sensor manifold were later designed and fabricated to provide a portable platform being developed towards a palm-sized system.

\section{VAPOR TEST MEASUREMENTS AND CONCLUSION}

The cantilever was coated with a custom NRL functionalized sorbent carbosilane polymer [4-5]. The tests were carried out by exposing the sensor to the nerve agent simulant, dimethylmethylphosphonate (DMMP) with isothermal conditions

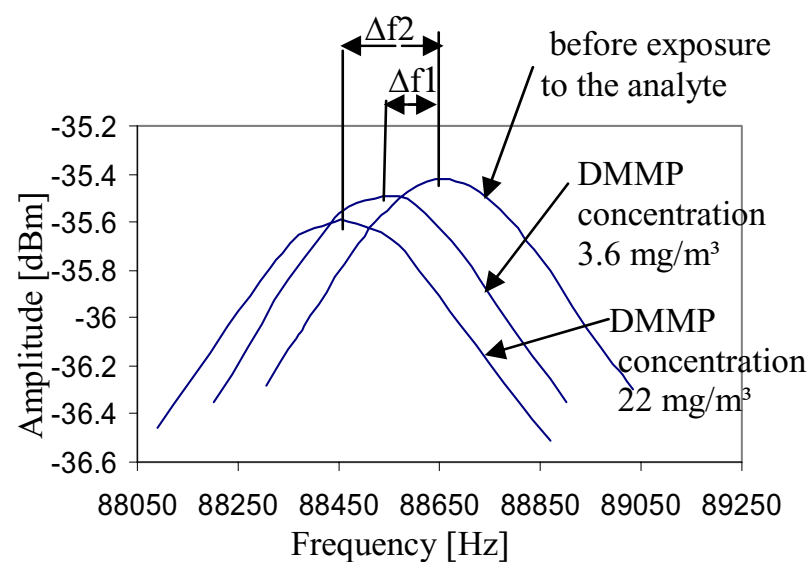

Figure 4. Cantilever response upon exposure to various DMMP concentrations. Frequency shifts are marked $\Delta f 1$ and $\Delta f 2$.

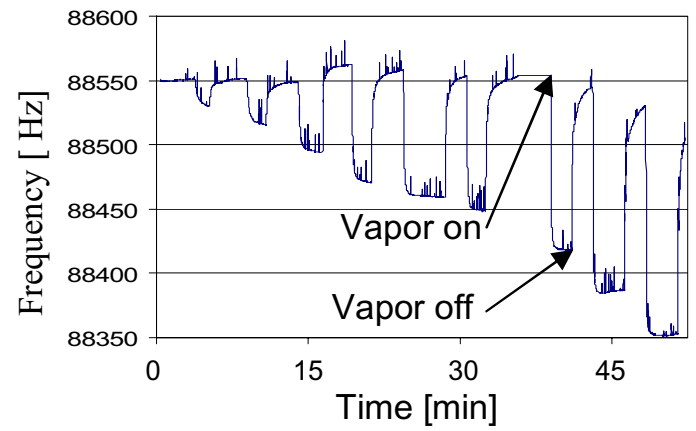

Figure 5. Frequency response to increasing DMMP concentration.

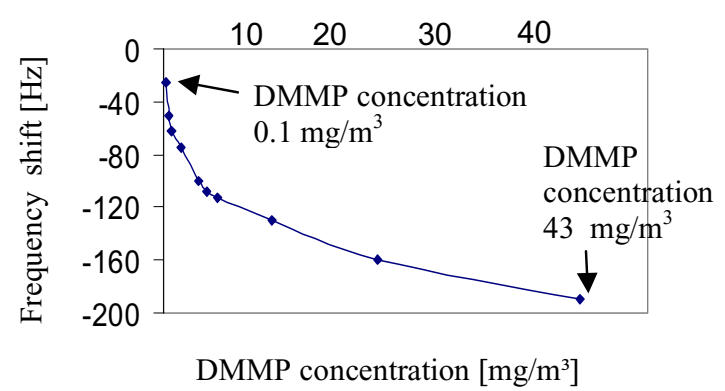

Figure 6. Cantilever response upon exposure to DMMP concentrations varying from 0.1 to $43 \mathrm{mg} / \mathrm{m}^{3}$.

at vapor concentrations which were ramped up and down from 0.1 $\mathrm{mg} / \mathrm{m}^{3}$ to $43 \mathrm{mg} / \mathrm{m}^{3}$ in a repeated fashion.

The cantilever sensor response, as a function of DMMP concentration, is shown in Fig. 4 and Fig. 5. The lowest DMMP concentration tested was $0.1 \mathrm{mg} / \mathrm{m}^{3}$ or $20 \mathrm{ppb}$, see Fig. $5 \& 6$. At this concentration, the signal frequency shift recorded was $20 \mathrm{~Hz}$, with an estimated signal noise level of about $1 \mathrm{~Hz}$. The sensor time constant to $90 \%$ of signal was approximately $10 \mathrm{~s}$.

These initial promising results were attained with a relatively simple design, fabricated in standard CMOS, which could offer an inexpensive option for production of a miniature chemical detector, with on chip electronics integrated to the cantilever.

\section{REFERENCES}

[1] R. A. McGill, M. H. Abraham, J. W. Grate, "Choosing polymer coatings for chemical sensors," CHEMTECH 24, pp. 27-37, 1994.

[2] H. Xie, L. Erdmann, X. Zhu, K. J. Gabriel, G. K. Fedder, "PostCMOS processing for high-aspect-ratio integrated silicon microstructures", J. Microelectromech. Sys., vol. 11, pp.93-101, 2002. [3] J. F. Vignola, X. Liu, S. F. Morse et al., Rev. Sci. Instrum Vol. 73, Nr.10, pp.3584 -3588, (2002).

[4] E. J. Houser, D. L. Simonson, J. L. Stepnowski, R. A. McGill "Linear and Hyperbranched Hydrogen Bond Acidic Poly(silylene methylene)s for Chemical Sensor Applications" PMSE Preprints 2003, 88, 548

[5] R. A. McGill, and E. J. Houser, "Linear chemoselective carbosilane polymers and methods for use in analytical and purification applications", U.S. Patent 6,660,230

\section{ACKNOWLEDGEMENTS}

The authors are indebted to Mr. John Petrella from the George Washington University for his contribution to the circuit design used to drive the resonant beam and his assistance. This work was supported in part by the Transportation Security Administration (TSA), and the Bureau of Alcohol, Tobacco, Firearms, and Explosives (ATF), and the Office of Naval Research (ONR). 